\title{
O SOFTWARE GEOGEBRA NO ENSINO DA FUNÇÃO QUADRÁTICA
}

\section{THE SOFTWARE GEOGEBRA IN THE TEACHING OF THE QUADRATIC FUNCTION}

\author{
Tamara Sued Pinheiro de Oliveira ${ }^{1}$; Dailton Cicerofram Souza Silva ${ }^{2}$, Ana Cristina de \\ Souza Lima ${ }^{3}$
}

\begin{abstract}
RESUMO
A tecnologia vem a cada dia redefinindo a maneira como são realizados determinados processos e com isso vem conquistando espaço nos mais diversos ambientes, fornecendo maneiras de se alcançar resultados mais precisos e solucionar problemas. No âmbito educacional, a utilização de tecnologia em sala de aula de matemática tem sido temática de diversas pesquisas. Diante desta realidade, traçamos como objetivo analisar a compreensão dos alunos no estudo da função quadrática por intermédio do GeoGebra. A realização dessa pesquisa é motivada por relatos de professores a respeito das dificuldades apresentadas pelos alunos das turmas de $1^{\circ}$ ano do ensino médio quanto ao estudo do conceito de função quadrática. A metodologia utilizada caracteriza-se como qualitativa com cunho exploratório. Dessa forma, buscamos contextualizar o desenvolvimento histórico do conceito de funções, as tecnologias no ensino da matemática e a importância do GeoGebra como ferramenta tecnológica de mediação na aprendizagem de conceitos relacionados à função quadrática. Os sujeitos da pesquisa são alunos de $1^{\circ}$ ano de uma escola de ensino médio da rede pública estadual da cidade de Acopiara-CE. Os instrumentos de coleta de dados foram o questionário de identificação dos sujeitos, atividade de sondagem de conhecimentos sobre a função quadrática e atividade pós-intervenção. Com base nos dados obtidos, comparamos os resultados das atividades antes e depois da utilização do GeoGebra, verificamos que a aprendizagem dos alunos com o auxílio do software GeoGebra se mostrou satisfatória, o conteúdo exposto se tornou mais concreto por meio da manipulação de atividades no GeoGebra, os alunos gostaram do programa e aprenderam mais facilmente os conceitos referente a função quadrática. Esperamos que este trabalho se torne útil para pesquisas futuras de
\end{abstract}

1 Pós-Graduanda no Ensino de Matemática pelo Instituto Nacional de Ensino (INE). E-mail: tamarasued@gmail.com.

(iD) ORCID iD: https://orcid.org/0000-0002-2201-5874.

2 Pós-Graduando no Ensino de Matemática pelo Instituto Nacional de Ensino (INE). Professor de Matemática vinculado à Secretaria de Estado de Educação do Ceará (SEDUC/CREDE 16), Iguatu, Ceará, Brasil. Endereço para correspondência: Rua 13 de maio, 55, Planalto, Iguatu, CE, Brasil, CEP: 63500-773. E-mail: dailton546@gmail.com.

(iD) ORCID iD: https://orcid.org/0000-0001-5962-4051.

3 Mestranda do Programa de Pós-Graduação em Ensino de Ciências e Educação Matemática da Universidade Estadual da Paraíba (UEPB). Professora do curso de Licenciatura em Matemática da Faculdade de Educação, Ciências e Letras de Iguatu, campus da Universidade Estadual do Ceará (UECE/FECLI), Iguatu, CE, Brasil. Endereço para correspondência: AV. DR. Dario Rabelo, S/N, Santo Antônio, Iguatu, CE, Brasil, CEP:63502-253. cris.acsl@gmail.com.

(iD) ORCID iD: https://orcid.org/0000-0003-2890-5533. 
professores e alunos interessados na área de tecnologia na educação, auxiliando-os na exploração deste conteúdo.

Palavras-chave: Tecnologia no Ensino; Função Quadrática; GeoGebra.

\begin{abstract}
Technology has been redefining the way certain processes are carried out every day and with this it has been conquering space in the most diverse environments, providing ways to achieve more accurate results and solve problems. In the educational field, the use of technology in the mathematics classroom has been the subject of several studies. Given this reality, we set out to analyze students' understanding of the quadratic function through GeoGebra. The realization of this research is motivated by teachers' reports about the difficulties presented by students of the 1st year of high school classes regarding the study of the concept of quadratic function. The methodology used is characterized as qualitative with an exploratory nature. Thus, we seek to contextualize the historical development of the concept of functions, technologies in the teaching of mathematics and the importance of GeoGebra as a technological tool of mediation in the learning of concepts related to the quadratic function. The research subjects are 1st year students from a public high school in the state public school in the city of Acopiara-CE. The instruments of data collection were the questionnaire of identification of the subjects, activity of polling knowledge about the quadratic function and post-intervention activity. Based on the data obtained, we compared the results of the activities before and after using GeoGebra, we found that the students' learning with the aid of the GeoGebra software proved to be satisfactory, the exposed content became more concrete through the manipulation of activities in GeoGebra, the students liked the program and learned the concepts related to the quadratic function more easily. We hope that this work will become useful for future research by teachers and students interested in the area of technology in education, assisting them in the exploration of this content.
\end{abstract}

Keywords: Teaching Technology; Quadratic function; GeoGebra. 


\section{Introdução}

Ensinar o conceito de função quadrática é uma tarefa que pode trazer dificuldade para os professores na hora de expor determinadas particularidades, como é o caso da interpretação gráfica. Por muitas vezes, a metodologia do professor de matemática se resume a utilizar o pincel e o quadro proporcionando ao aluno uma quantidade limitada de informações.

Sabendo da importância em buscar recursos pedagógicos capazes de auxiliar a prática docente na busca pela superação das dificuldades dos alunos perante o ensino da matemática, esta pesquisa compreende a aplicabilidade do uso de software matemático GeoGebra como ferramenta metodológica para a sala de aula. Tendo por objetivo analisar a compreensão dos alunos no estudo da função quadrática por intermédio do GeoGebra.

A motivação para abordagem deste tema surge a partir de relatos de professores das turmas $1^{\circ}$ de ano do ensino médio de uma escola estadual na cidade de AcopiaraCeará, durante os planejamentos de área. Tais professores relataram as dificuldades dos alunos em compreender alguns conceitos relacionados à função quadrática.

Para ajudar os alunos a compreender com mais facilidade alguns conceitos geométricos, o professor pode recorrer a algumas ferramentas digitais, visto que, "as tecnologias digitais móveis desafiam as instituições a saírem do ensino tradicional, em que o professor é o centro, para uma aprendizagem mais participativa e integrada" (MORAN, 2013, p. 57). Nessa perspectiva, para o ensino de função quadrática o professor pode fazer uso do GeoGebra, uma ferramenta de fácil acesso e gratuita.

O GeoGebra auxilia na visualização para uma explicação mais detalhada dos conceitos gráficos da função em questão, e seu uso se justifica pela quantidade e a variabilidade de ferramentas tecnológicas às quais os alunos se expõem diariamente. Expor o gráfico da função quadrática com este software pode contribuir para a compreensão do aluno, pois "trazer uma mídia informática para a sala de aula significa abrir a possibilidade de os alunos falarem sobre suas experiências e curiosidades nesta área" (BORBA; PENTEADO, 2012, p. 12).

Segundo orientação da competência geral 5 da Base Nacional Comum Curricular (BNCC) sobre o uso de tecnologias o professor deve, 
práticas sociais (incluindo as escolares) para se comunicar, acessar e disseminar informações, produzir conhecimentos, resolver problemas e exercer protagonismo e autoria na vida pessoal e coletiva (BRASIL, 2017, p.9).

Delineamos na sequência deste artigo, aspectos históricos do conceito de função, em seguida uma abordagem da tecnologia no ensino de matemática e também será exposta a maneira como utilizar o GeoGebra para se trabalhar os conceitos de função quadrática.

Na metodologia temos o desenvolvimento da pesquisa, onde é descrito o lócus da pesquisa, os sujeitos e a coleta de dados; e finalizamos com a análise dos dados e a discussão dos resultados, com a comparação entre as atividades realizadas e os efeitos da intervenção com o GeoGebra em aulas de matemática.

\section{Breve Histórico do Desenvolvimento de Função}

Sempre que nos deparamos com determinados assuntos matemáticos é comum surgir o questionamento de como esse conceito foi formado e quem o desenvolveu. Para a construção do conceito de função ideias primitivas já surgiam na Antiguidade, Roque e Carvalho (2012, p. 264) afirmam que "tabelas babilônias e egípcias já pressupunham, de alguma forma, a ideia de função, uma vez que se tratavam justamente de registros de correspondência".

Podemos citar a contribuição da civilização grega em que a matemática e astronomia estavam interligadas, o que nos faz acreditar que era perceptível aos gregos uma ideia de dependência funcional, construindo tábuas onde registravam a posição do sol, da lua e dos planetas observando que a variação da posição desses astros acontecia de forma contínua e periódica (BOYER, 1996).

Mas a ideia de variação, que está relacionada ao conceito de função que temos hoje, ainda não estava presente nessas civilizações, pois esta relação acontece após os estudos de Galileu Galilei (ROQUE; CARVALHO, 2012). Como destaca Maciel Júnior (2019, p. 30), "foi a partir dos estudos de Galileu que causa e efeito puderam ser expressos e verificados quantitativamente, isto é, vinculando causa e efeito, de modo a gerar o que posteriormente seria definido como variável dependente”.

Não podemos deixar de mencionar René Descartes que começou a tratar de maneira algébrica problemas geométricos, “(...) as grandes inovações foram a associação 
de curvas a equações algébricas e o uso de um sistema de coordenadas para relacionar as variáveis envolvidas naquelas equações" (BOTELHO; REZENDE, 2007, p. 68). Com isto, foi possível a associação entre curvas e equações permitindo uma interpretação geométrica para a álgebra.

Um trabalho que tratasse de maneira específica às funções foi desenvolvido por Leibniz e Newton com o uso das séries infinitas, no século XVIII, "a função foi, assim, definida como uma expressão analítica composta de um modo qualquer de quantidades constantes e variáveis" (ROQUE; CARVALHO, 2012, p. 296).

Diante de vários trabalhos, é possível perceber que o conceito de função sofreu muitas alterações, fruto do trabalho de diversos povos em diferentes civilizações. Atualmente, livros didáticos do Ensino Médio definem função como, "dados dois conjuntos $\mathrm{A}$ e $\mathrm{B}^{(*)}$, não vazios, uma relação $\mathrm{f}$ de $\mathrm{A}$ em $\mathrm{B}$ recebe o nome de aplicação de A em $B$ ou função definida de A com imagens em $B$ se, e somente se, para todo $\mathrm{x} \in \mathrm{A}$ existe só um y $\boldsymbol{\epsilon}$ B tal que (x, y) $\boldsymbol{\epsilon}$ f' (IEZZI; MURAKAMI, 2004, p. 81). Os livros trazem exemplos de funções contextualizando com exemplos do cotidiano e relacionado aos conceitos de produto cartesiano e relações.

De acordo com Lima (2012), no que tange à função quadrática, sua origem está ligada à resolução de equações do segundo grau desde a época dos babilônios, que em seus textos cuneiformes, tratava de encontrar dois números conhecendo sua soma $s$ e seu produto $p$. "Os números procurados são as raízes da equação do segundo grau $x^{2}-s x$ $+p=0 ”($ LIMA, 2012, p. 119)

Segundo Lima (2012, p.114- p.125), "uma função $f: \mathcal{R} \rightarrow \mathcal{R}$ chama-se quadrática quando existem números reais $a, b, c$ com $a \neq 0$ tais $f(x)=a x^{2}+b x+c$ que para todo $x$ $\in \mathcal{R}$. O gráfico de uma função quadrática é uma parábola”.

A importância do estudo de função não é restrita apenas aos interesses da matemática, mas está presente em outras ciências, como por exemplo, na física, na biologia, na química, na contabilidade e na engenharia civil. Por isso, é importante para o aluno conhecer o percurso histórico e compreender os conceitos relacionados a este conteúdo. Nessa perspectiva, cabe ao professor utilizar estratégias pedagógicas que facilitem o entendimento para o aluno. A inserção do GeoGebra, como recurso didático e tecnológico, no ensino de funções pode contribuir para a melhoria da aprendizagem dos alunos. 


\section{Tecnologia no ensino de matemática}

Muitos dos desenvolvimentos tecnológicos impulsionaram mudanças na sociedade. Sabemos que durante todo o seu desenvolvimento a humanidade encontra-se em constante transformação em seus hábitos e maneiras de realizar tarefas; aprendeu a transformar o meio em que vive e a adaptar-se às mais diversas situações.

A introdução dos meios tecnológicos na educação brasileira ocorreu por volta de 1970, do século XX, com a implementação de alguns programas através da Secretaria de Educação à Distância (SEED) do Ministério da Educação. Esses programas visavam inovar e com isso promover uma evolução no ensino. Um dos primeiros programas a serem implementados foi o Programa Nacional de Tecnologia Educacional (PROINFO).

Com essa implementação pontos de vista diferentes começaram a aparecer, uma das primeiras indagações eram se as escolas, professores e gestores estavam preparados para a utilização da tecnologia. No entanto, muitos se mostravam contra, por medo de serem trocados por máquinas, embora o sentido de utilizar a tecnologia não seja substituir o professor e nem diminuir o esforço dos alunos.

Muitas escolas adquiriram computadores, mas os usavam apenas para atividades de pesquisa ou para fins burocráticos, ou seja, sem nenhuma mudança pedagógica. Sabemos que a tecnologia precisa estar inserida em sala de aula, auxiliando na prática pedagógica.

\footnotetext{
A educação no mundo de hoje tende a ser tecnológica, por isso, exige entendimento e interpretação, tanto dos professores quanto dos alunos em relação a essas novas tecnologias. Através do uso da tecnologia no ambiente escolar, ficam claros os diversos sentimentos em relação a postura dos professores frente a novos desafios, como a satisfação de estar participando de uma realidade tecnológica ou a ansiedade por enfrentar novas mudanças. E em relação aos alunos também ocorrem transformações, pois passam a ficar mais motivados para estudar e aprender, e as aulas não ficam tão expositivas (THOALDO, 2010, p.25).
}

Com o advento da internet, foi possibilitado que as informações passassem a circular com maior facilidade e extrema agilidade. A partir do seu uso como abundante meio de pesquisa, o acesso fácil a softwares gratuitos desenvolvidos para uso em práticas pedagógicas tornou-se viável. A partir desse movimento, é que começou a inserção de aplicativos na educação pautados nas contribuições para a aprendizagem dos alunos. 
Nessa perspectiva, utilizar software educacional como ferramenta pedagógica para o ensino da matemática, auxilia o docente em suas atividades didáticas, uma vez que a matemática lida sempre com a abstração.

Assim, todo conhecimento matemático deve ser estruturado de forma que os alunos possam edificar suas concepções visto que,

\begin{abstract}
O professor, a quem cabe a tarefa de ensinar, deixa de ser um mero transmissor de conhecimentos descontextualizados, para se tornar um catalisador do conhecimento, mediador do processo de aprendizagem, responsável por preparar as condições para que ocorra a aprendizagem. (VIDAL; MAIA, 2002, p. 123).
\end{abstract}

Alguns conceitos matemáticos a serem abordados em sala de aula podem trazer de certa forma, um nível maior de dificuldade para alguns professores no sentido de tornar a aula interativa e podem propiciar aos alunos uma maior participação. Como exemplo o conteúdo de função quadrática: durante a explicação, o professor deve traçar gráficos no quadro para auxiliar a compreensão de determinado fenômeno pelos alunos, como a trajetória de um projétil; mas, encontramos problemas quando é o momento de os alunos fazerem o mesmo, pois para muitos o desenho no quadro não indica o movimento desejado, se tornando assim apenas uma figura estática e sem possibilidades de manipulação.

Nesse sentido, a BNCC ressalta importância da tecnologia para os estudantes:

O uso de tecnologias possibilita aos estudantes alternativas de experiências variadas e facilitadoras de aprendizagens que reforçam a capacidade de raciocinar logicamente, formular e testar conjecturas, avaliar a validade de raciocínios e construir argumentações (BRASIL, 2017, p.9).

Com isso, o GeoGebra pode ser considerado uma ferramenta facilitadora para o ensino de funções quadráticas. A utilização das ferramentas do GeoGebra proporciona mais praticidade e dinamicidade ao trabalhar com funções matemáticas, especificamente a função quadrática. Essas ferramentas passam a servir de apoio para o professor ao facilitar a explicação do conteúdo para a turma, permitindo que os alunos possam formalizar de forma concreta o objeto de estudo. 


\section{GeoGebra no ensino da função quadrática}

De acordo com o site oficial do aplicativo, o GeoGebra é considerado um software de matemática dinâmica, gratuito e multiplataforma para todos os níveis de ensino, que combina geometria, álgebra, tabelas, gráficos, estatística e cálculo numa única aplicação. Está disponível online e pode ser baixado no endereço eletrônico: http://www.GeoGebra.org. Depois de instalado não há a necessidade de está conectado a internet para manuseá-lo.

A utilização deste software possibilita ao professor de matemática uma melhor forma de trabalhar a construção de gráficos de funções, além de proporcionar uma aula interativa com participação efetiva do aluno e contribuir para um desenvolvimento cognitivo concreto do discente.

Através do GeoGebra, é possível em apenas uma única tela mostrar o comportamento do gráfico de determinada função e também movimentá-lo, trazendo para o aluno uma visão mais concreta do que está acontecendo.

O grande diferencial deste recurso é a possibilidade de manipulação tanto para o professor quanto para os alunos. Tomamos como exemplo o estudo das funções, o professor pode fazer o gráfico da função no quadro e dizer para os alunos que esse mesmo gráfico pode mudar à medida que os pontos variam, mas sem fazer outro gráfico no quadro não é tão perceptível aos alunos a visualização do que foi exposto. Dessa maneira, o processo acaba se tornando uma repetição de desenhos de gráficos que os alunos refazem no caderno sem muitas vezes não terem conseguido visualizar realmente o que está acontecendo.

Souza diz que esta ferramenta tecnológica [...] "facilita e dinamiza o processo de aprendizagem dos alunos de forma que, ao passarem informações para o software, recebem instantaneamente respostas que correlacionam expressões algébricas com as suas respectivas representações gráficas" (SOUZA, 2012, p. 91).

No âmbito de estudos sobre funções quadráticas e a utilização do GeoGebra, Souza (2013) conclui que a representação gráfica das propriedades era compreendida facilmente pelos alunos, pois o conhecimento era adquirido por eles próprios por intermédio da experimentação. 
Para trabalhar o conteúdo de função quadrática, o GeoGebra fornece meios para desenvolvê-lo de forma mais clara, fazendo uma maior aproximação com a realidade do aluno.

\section{Metodologia}

Este estudo é de cunho qualitativo, uma vez que, como assinala André (2002, p. 33), a abordagem qualitativa consiste em "um aprendizado de pesquisa da própria realidade para conhecê-la melhor e poder vir a atuar mais eficazmente sobre ela, transformando-a".

Os sujeitos desta pesquisa são 40 alunos de uma turma do $1^{\circ}$ ano do ensino médio, do turno manhã, em uma escola pública de ensino médio situada no município de Acopiara-CE. A instituição de ensino onde a pesquisa foi desenvolvida está sob jurisdição da Secretaria Estadual da Educação do Ceará, através da $16^{\text {a }}$ Coordenadoria Regional de Desenvolvimento da Educação (CREDE 16). O trabalho realizado nesta pesquisa aconteceu nos meses de março e abril de 2019.

Nessa escola, na época da pesquisa, existiam sete turmas de primeiro ano do ensino médio. A escolha da turma deu-se pela compatibilidade de horário do pesquisador em relação às aulas de matemática. Uma vez que o pesquisador leciona matemática nas turmas do segundo ano do ensino médio e teria que acompanhar por dois meses as aulas do professor titular para realizar a pesquisa.

Tomando por base o relato feito pelo o professor titular a respeito das dificuldades dos alunos no estudo da função quadrática, surge a inquietação em identificar quais seriam as contribuições que o GeoGebra traria para o aprendizado dos alunos, uma vez que este software possibilita a dinamicidade durante a explicação dos conteúdos, como já citado anteriormente.

O desenvolvimento da pesquisa foi organizado em quatro etapas: aplicação do questionário de identificação dos sujeitos; aplicação do questionário de sondagem de conhecimentos sobre função quadrática; intervenção com o GeoGebra e aplicação da atividade de avaliação de conhecimento pós intervenção metodológica com o GeoGebra. 


\section{Análise dos dados e discussão dos resultados}

Para obter uma melhor análise dos dados, é necessário conhecer os sujeitos que fazem parte da pesquisa. Para isso, os 40 alunos que participaram das atividades desenvolvidas ao longo dessa pesquisa foram submetidos a um questionário de identificação dos sujeitos. Os discentes estão na faixa etária entre 15 e 17 anos, 11 são do gênero masculino e 29 são do gênero feminino. Por questões éticas a identidade dos participantes não será revelada.

Em relação ao contato com as tecnologias, constata-se que: $82,5 \%$ dos alunos afirmam que acompanham os avanços tecnológicos e 17,5\% dos alunos responderam que não acompanhavam. Esse fato se justifica pela proximidade que os alunos têm com a tecnologia no seu dia a dia.

A segunda pergunta visa detectar se os discentes utilizam algum recurso tecnológico durante seus estudos: 97,5\% dos alunos responderam que utilizavam algum recurso e 2,5\% responderam que não. Em seguida, foram questionados se utilizavam o laboratório de informática de sua escola para realização de pesquisas escolares: 95\% dos alunos responderam que fazem uso do laboratório em seus estudos para realização de pesquisas e $5 \%$ responderam que não.

A quarta pergunta versava sobre a utilização de ferramentas tecnológicas em aulas de matemática: $82,5 \%$ dos alunos afirmaram que o professor não faz uso de ferramentas tecnológicas durante suas aulas e 17,5\% afirmaram que o docente as utiliza. A quinta pergunta questionava se os alunos acreditavam que a tecnologia poderia ajudar no aprendizado de matemática: $95 \%$ dos alunos acreditam que pode ajudar e 5\% afirmam que não poderia ajudar.

A sexta e última pergunta relacionava alguns softwares voltados para o ensino de matemática e o nível de conhecimento que os alunos tinham a respeito destes softwares. Com base nas respostas, concluímos que $87,5 \%$ da turma não conhece ou não foram apresentados a nenhum software para o ensino de matemática, 2,5\% conhece ou foram apresentados ao Winplot, 2,5\% também conhece ou já teve contato com o Poly e que 7,5\% da turma conhece ou já teve contato com o GeoGebra.

$\mathrm{Na}$ segunda etapa, aplicou-se um questionário, composto por cinco questões, buscando identificar os conhecimentos dos alunos sobre a função quadrática. A análise das respostas será apresentada a seguir. 
Na primeira questão, os alunos precisavam reconhecer os valores dos parâmetros $a, b$ e $c$ de uma função quadrática. Os resultados obtidos mostram que $20 \%$ dos alunos da turma conseguiram reconhecer os valores desses parâmetros e $80 \%$ da turma não conseguiram reconhecer os referidos valores, resultando em uma quantidade elevada de alunos que não dominam esse conhecimento.

Na segunda questão, foi proposto aos alunos construírem o gráfico da função $f(x)$ $=x^{2}+4$, identificando as raízes (ou zeros) da função quadrática, a concavidade do gráfico da função, o ponto máximo ou mínimo. Com base nos resultados obtidos nesta questão, observamos que 5\% dos alunos conseguiram resolver corretamente a questão e $95 \%$ não conseguiram resolver ou deixaram de resolver. Observamos uma quantidade elevada de alunos que não dominam os conceitos abordados na questão.

$\mathrm{Na}$ terceira questão, foram dadas quatro funções para os alunos construírem os respectivos gráficos comparando as similaridades e diferenças entre eles. Temos que 5\% dos alunos conseguiram responder de forma correta a questão e que $95 \%$ não conseguiram responder ou deixaram de responder.

A questão quatro avaliava a capacidade de o aluno determinar a imagem de um número dada uma função quadrática: $47,5 \%$ da turma respondeu de forma correta a questão e 52,5\% não responderam corretamente ou não responderam à questão.

A quinta questão avaliava no aluno a capacidade de identificar e criar funções quadráticas variando os valores do parâmetro $a$, percebemos que $10 \%$ dos alunos responderam de forma correta e $90 \%$ dos alunos não resolveram corretamente ou deixaram de responder.

A terceira etapa da pesquisa consiste na apresentação do GeoGebra como uma ferramenta auxiliar para o estudo da função quadrática. Optou-se pela realização dessa etapa em dois momentos, o primeiro em sala de aula e o segundo no laboratório de informática da escola. Durante duas semanas, às terças-feiras, no tempo de duas horas aulas semanais, iniciamos em sala de aula uma explanação do conteúdo de função quadrática e depois a apresentação do GeoGebra para os alunos. A versão utilizada foi o GeoGebra Classic online, sendo o layout das ferramentas disponibilizadas na tela do GeoGebra semelhante ao layout do aplicativo GeoGebra, que pode ser instalado no aparelho celular (Figura 1). 
Figura 1 - Tela inicial do GeoGebra Classic

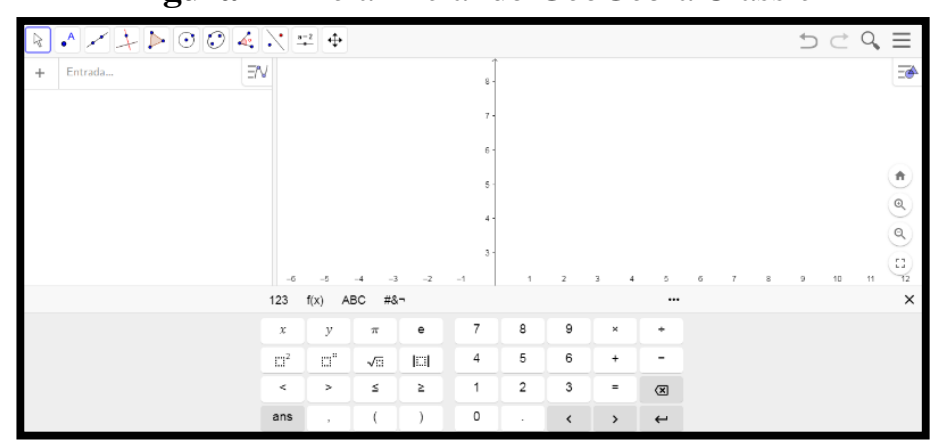

Fonte: Elaborado pelos autores

Utilizamos o recurso de notebook e Datashow, foram feitas as projeções no quadro branco para que todos os alunos pudessem acompanhar o que seria abordado. Explicamos como fazer a instalação do software, considerando que existe a possibilidade de instalálo em smartphones. Assim, aqueles alunos que não tenham computadores em casa podem acessá-lo pelo celular. A apresentação aconteceu de forma a chamar a atenção dos alunos e, com isso, foram explanados todos os benefícios que este recurso pode trazer para o aprendizado dos discentes.

Durante esta parte da intervenção, foi apresentado aos alunos um tutorial sobre o GeoGebra, sua interface e os seus principais comandos como a tela inicial do GeoGebra (Figura 1), onde se podem visualizar barras de menus e ferramentas, caixa de entrada, janela de álgebra e visualização.

O segundo momento dessa etapa, acontece no laboratório de informática da escola. Durante duas semanas, a turma foi dividida em dois grupos de vinte alunos, um grupo ficava na sala de aula com o professor titular de matemática e o outro era direcionado ao laboratório, a fim de praticarem as atividades com o auxílio do GeoGebra, acompanhados pelo pesquisador. A escolha em fazer a divisão em grupos deve-se ao fato de o laboratório de informática da escola dispor de poucos computadores funcionando.

Nesse momento da pesquisa, os alunos tiveram contato direto com o GeoGebra, aprenderam a manuseá-lo, inserirem as funções na caixa de entrada, analisarem as funções construídas e marcarem pontos no gráfico. Em seguida, foi feito o estudo dos conceitos de função quadrática por meio do mesmo, principalmente os conceitos relacionados ao gráfico da função quadrática e os conceitos abordados no teste anterior à intervenção, com a finalidade de que os discentes aprendessem a utilizar a ferramenta para as próximas atividades. Os alunos aprenderam com facilidade a utilizar o GeoGebra, 
relataram que a fácil assimilação se deu pela semelhança entre o teclado do software e do aparelho celular.

As atividades realizadas estavam ligadas ao gráfico da função quadrática, sua construção e interpretação. Os alunos eram livres para explorar esse conteúdo. Por muitas vezes relataram situações que presenciaram em sala de aula, mas que não tinham sido compreendidas e com uso do software tiveram uma melhor compreensão, como no caso da relação entre o discriminante Delta e a quantidade de raízes por intermédio da análise do gráfico da função.

Após esse período de intervenção com o auxílio do GeoGebra, na quarta etapa foi aplicado uma atividade semelhante ao teste de sondagem (segunda etapa), buscando identificar os conhecimentos dos alunos em relação às funções quadráticas, e assim analisar as contribuições que o uso do software pode trazer para o ensino da matemática. As questões propostas mantêm o nível de conhecimento e os mesmos assuntos que foram abordados na atividade de sondagem.

As atividades eram semelhantes no nível de conhecimento exigido em cada questão, mas com os dados das questões diferentes para que a avaliação do processo de aquisição do conhecimento apresentasse resultados reais. Todo o processo da pesquisa foi proposto para acontecer da forma mais imparcial possível e os resultados apresentados por ela serem de fato dados concretos.

Ao fazer um comparativo entre a atividade de sondagem e a atividade pósintervenção, percebemos que o aumento na quantidade de alunos que responderam corretamente a atividade pós-intervenção com o GeoGebra foi significativamente grande, principalmente naquelas que envolviam a análise do gráfico da função, conceito este que foi constatado por meio do teste de sondagem e que apresentou maiores dificuldades dos alunos. (Figura 2). 
Figura 2 - Comparativo entre os testes de sondagem e pós-intervenção.

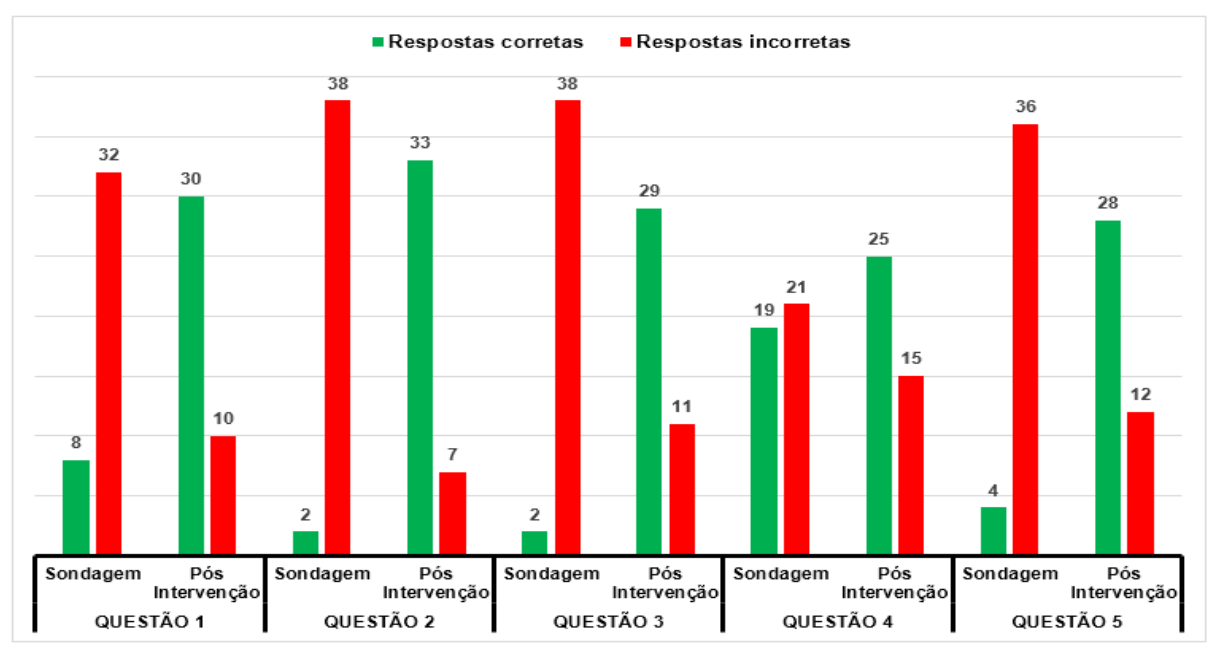

Fonte: Elaborado pelos autores

Pelas análises, percebe-se que o GeoGebra permite o ensino de função quadrática de forma concreta. Durante as manipulações, os alunos compreenderam mais facilmente que ao alterar os parâmetros das funções acontecem as variações da função (crescimento, concavidade e extremos), que mudam de acordo com os parâmetros.

Ao explicar o conteúdo fazendo a exposição no quadro, desenhando os gráficos, a aula pode ser cansativa e desestimulante para o aluno; já com o auxílio do GeoGebra é possível visualizar a movimentação do gráfico, alterando um dos coeficientes sem abandonar a função original.

Assim, corroboramos com Lima (2013, p. 56) ao afirmar que, "[...] As vantagens desta manipulação ficam evidentes como, por exemplo, com o fato de podermos realizar várias manipulações em pouco tempo, diferentemente de um gráfico construído com lápis e papel".

Dessa forma, é importante que o professor utilize cada vez mais ferramentas digitais no ensino de matemática e que busque se aperfeiçoar para utilizar essas ferramentas com domínio. Assim, o ensino de matemática acontecerá de forma dinâmica e, consequentemente, despertará o interesse no aluno.

\section{Considerações finais}

A tecnologia tem um potencial muito grande quando se trata em contribuir para a educação. Ao ser aplicado com uma finalidade o GeoGebra se mostrou eficiente em 
contribuir para o aprendizado dos alunos; forneceu visualização de gráficos facilitando a sua interpretação e o reconhecimento de alguns pontos notáveis; sem mencionar o fato de motivar os alunos a serem autônomos e, por meio da experimentação, indica contribuições para a aprendizagem.

Em linhas gerais, o software proporcionou aos alunos uma oportunidade de observar o conceito de função quadrática de forma diferente, um fato relevante foi observado na atividade de sondagem de conhecimentos, a maioria dos alunos se mostrou desmotivados em realizar a atividade. Com a intervenção do GeoGebra foi possível identificar maior interesse dos alunos e melhor compreensão do conteúdo explanado: construir o gráfico de funções quadráticas, identificar os coeficientes compreendendo o significado geométrico; identificar a concavidade, quantidade de raízes e os pontos de máximo e mínimo da função.

Os resultados obtidos com a pesquisa foram satisfatórios no aspecto de aprendizagem dos alunos, o que leva a conclusão de que a intervenção com o GeoGebra, como recurso pedagógico, contribui significativamente para o ensino e para a aprendizagem do conceito de função quadrática, principalmente no que diz respeito às suas interpretações gráficas.

\section{Referências}

ANDRÉ, M. E. D. Etnografia da Prática Escolar. 8. ed. Campinas: Papirus, 2002.

BORBA, M. C, PENTEADO, M. G. Informática e Educação Matemática. 5. ed. Belo Horizonte: Autêntica editora, 2012.

BOTELHO, L.; REZENDE, W. M. Um breve histórico do conceito de função. Caderno Dá-Licença, Niterói, v. 6, p.64-75, 2007.

BOYER, C. B. História da Matemática. Tradução de Elza F. Gomide. 2. ed. São Paulo: Editora Blücher, 1996.

BRASIL. Base Nacional Comum Curricular: Educação é base. Ministério da Educação. Brasília: MEC, 2017. Disponível em: http://basenacionalcomum.mec.gov.br/images/BNCC EI EF 110518 versaofinal site. pdf. Acesso em: 16 de agosto de 2019.

IEZZI, G.; MURAKAMI, N. Fundamentos de Matemática Elementar. 7. ed. São Paulo: atual, 2004. 
LIMA, C. E. O. A utilização do software GeoGebra como ferramenta para o ensino de funções. 2013. 64 f. Dissertação (Mestrado em Matemática em Rede Nacional) Centro de Ciências, Universidade Federal do Ceará, Fortaleza, 2013. Disponível: http://www.repositorio.ufc.br/bitstream/riufc/5815/1/2013 dis ceolima.pdf. Acesso em $23 / 02 / 2021$

LIMA, E. L. et al. A Matemática do Ensino Médio. 10. ed. Rio de Janeiro: SBM,2012.

MACIEL JUNIOR, J. M. A. Um estudo sobre o uso do software GeoGebra para o ensino de matemática: uma proposta de sequência didática para o conteúdo de função quadrática. 2019. 56 f. Trabalho de Conclusão de Curso (Graduação em 2019) Universidade Estadual do Ceará, 2019. Disponível em: http://siduece.uece.br/siduece/trabalhoAcademicoPublico.jsf?id=88301 Acesso em: 25 de fevereiro de 2021

MORAN, J. M; MASETTO, M; BEHRENS, M. Novas Tecnologias e Mediação Pedagógica. São Paulo: Papirus Editora, 2013.

ROQUE, T; CARVALHO, J.B.P. Tópicos de História da Matemática. Rio de Janeiro: SBM, 2012.

SOUZA, R. M. O uso do GeoGebra no ensino da função quadrática. 77 f.

Dissertação (Mestrado Profissional em Matemática em Rede Nacional) — Universidade Federal do Oeste do Pará-UFOPA, Santarém, 2013. Disponível em: https://sca.profmatsbm.org.br/sca_v2/get_tcc3.php?id=31386 . Acesso em: 09 mar. 2019.

SOUZA, F. A. L. de. O uso do software GeoGebra como ferramenta pedagógica no estudo de funções quadráticas em turmas de $9^{\circ}$ ano do ensino fundamental do CMF. 2012. 108 p. Dissertação (Mestrado profissional em ensino de ciências e matemática) - Universidade Federal do Ceará - UFC, Fortaleza, 2012.

THOALDO, D.L.P.B. (2010) O uso da tecnologia em sala de aula. Trabalho de Monografia apresentado na pós-graduação em Gestão Pedagógica da Universidade Tuiuti do Paraná 1: 1- 35.

VIDAL, E. M.; MAIA, G. L. S. B. Educação, informática e professores. Fortaleza: Edições Demócrito Rocha, 2002.

Recebido em: 27 / 02 / 2021

Aprovado em: 17 / 04 / 2021 